\title{
Understanding Loudness variations for aircraft Landing procedures at Arlanda (ULLA)
}

\author{
Anders Johansson and Karl Bolin \\ KTH/MWL, Center for Sustainable Aviation Stockholm, Sweden \\ E-mail: aebjo@kth.se, kbolin@kth.se
}

\begin{abstract}
As of today air traffic is increasing at Arlanda airport, resulting in higher noise levels. This project has been launched to investigate the possibilities of optimizing flight procedures for a quieter approach. A network of acoustic measurement stations has been developed to measure single aircraft pass-by noise at ground level. The pass-by noise levels have been evaluated together with flight trajectory's and the current meteorological conditions in order to investigate how operational procedures impact the noise distributions on ground. This paper presents the result of from a snap-shot of measurements that show variations in generated noise levels for similar approach routes and weather conditions. Difference as large as $10 \mathrm{~dB}$ was found between, pass-by's performed by the same individual aircraft. The reduced velocity for the quieter flight, stands out as the major factor to reduce noise levels.
\end{abstract}

Keywords: Acoustical measurements, Aircraft noise

\section{Introduction}

Over the last few decades there has been a global increase in air traffic and Arlanda is no exception to the trend, rather the opposite, with far-reaching plans to expand and build additional runways to accommodate the increase in air traffic. This new situation urges the importance of investigating and evaluating the noise issues surrounding Arlanda in order to maintain a sound relationship with its nearby residents.

An epidemiological survey of 1240 residents around Arlanda carried out by KTH / MWL 2009-2010 in the EU project COSMA [1] provides that those who live near the approach routes are more disturbed than those who live near the departure routes. Of interest is therefor to perform long-term measurements around Arlanda to investigate how the sound sources vary during the approach and if this can be a basis for sound optimized approach procedures?

Aircraft noise are generated by both the engines and the aircraft body. The approximate relationship between engine and airframe noise at landing is about $50-50 \%$ with a slight overweight for sound generated by the aircraft body which will be even more accentuated with the ongoing development of more quiet engines. This means that noise from other components of the aircraft has increased in importance at landing [2]. It is therefore considered a priority to measure sound for different landing procedures and meteorological conditions to analyze if there are differences in noise distributions related to flight operational variables.

Dose-response relationships between noise level and perceived annoyance for nearby residents are well established [3], but behind these connections it appears to be a significant variation in annoyance at the same noise level. Thus, annoyance from aircraft cannot only be explained by the noise levels the residents experience. The connection between sound and noise have to be explained by more variables and the sound quality is probably part of the explanation. Thus, there is a need to evaluate not only the sound level from different flight operations, but also how other sound aspects i.e. the sound quality is affected.

Considering the above aspects of aircraft noise and its components, sound measurements are of central importance for obtaining a detailed view of the noise situation around Arlanda approach routes. In order for this analysis to be possible, coordinated measurements are needed for several positions and preferably over a long period of time to ensure a solid statistical basis. Measurements of this kind generally involve high costs due to expensive measuring equipment. However, the development of Single Board Computers (SBC) together with cheap USB-interface microphones have enabled the composition of measurement equipment to a 
considerable reduced cost. In this project, which is part of the Centre for Sustainable Aviation at KTH (CSA), five autonomous measurement stations have been built to form a network of measurement stations. The network covers an area equal to a circle of $2 \mathrm{~km}$ radius and is deployed to cover part of the approach to runway 26 to capture time and spatial variations in the sound field. Together with meteorological data and Secondary Surveillance Radar (SSR) the measurements are analyzed to investigate how sound field variations are related to the flight operational procedures.

\section{Method}

To enable the analysis, three sets of data is needed: the Aircraft trajectory, the current meteorological condition and the noise level on ground. How these are gathered is described below.

\subsection{Aircraft trajectory}

The aircraft trajectory is downloaded live from the online flight surveillance site at OpenSky Network [4]. The main technologies behind the OpenSky Network are the Automatic Dependent Surveillance-Broadcast (ADS-B) and Mode S which is a modern method for SSR. With this technology the aircraft determines its position via GPS and then periodically broadcasts it together with other aircraft information. The main difference to older systems of SSR is that no interrogation signal is needed and that the information is broadcast over the $1090 \mathrm{MHz}$ radio frequency channel, making it publicly accessible. The OpenSky Network compiles ADS-B data into a set of variables which is updated in approximate real time every 5 seconds. The most important of these variables is data for position, altitude, speed and the ICAO24 address, which enables the identification of the aircraft. While the complete trajectory and movement of the aircraft is given by the ABS-B data there are no directly available variables that give information about the flight configuration i.e. thrust and flap settings, extension of landing gear, weight of aircraft etc. To directly collect such data access to the Flight Data Recorder (FDR) would be needed. There are however methods to estimate some of these parameters from the ADS-B data, for example the thrust setting, though this procedure is not covered in this paper.

\subsection{Meteorological data}

The meteorological data used in this project is provided from the numerical forecasting model AROME [5]. The model describes weather developments in time and space, in a grid of $2.5 \times 2.5 \mathrm{~km}$ squares where the atmosphere has been divided into 65 levels in the vertical direction. The forecast is based on what the weather looks like at a certain time, the socalled initial state. Every third hour the forecast is updated with an analysis of the current weather situation. In this way, the forecast is modified to better match current observations. The model has been developed within an international collaboration involving a large number of European countries. The AROME analysis is performed over an area that covers Scandinavia, the northern part of the continent and the Baltic States.

\subsubsection{Acoustic measurement stations}

Acoustic measurements of aircraft pass-by's are warranted for an extended period of time, preferably covering the seasonal changes of the year and both day and night. To meet this demand, several autonomous measurement stations has been built, see fig. 1 .

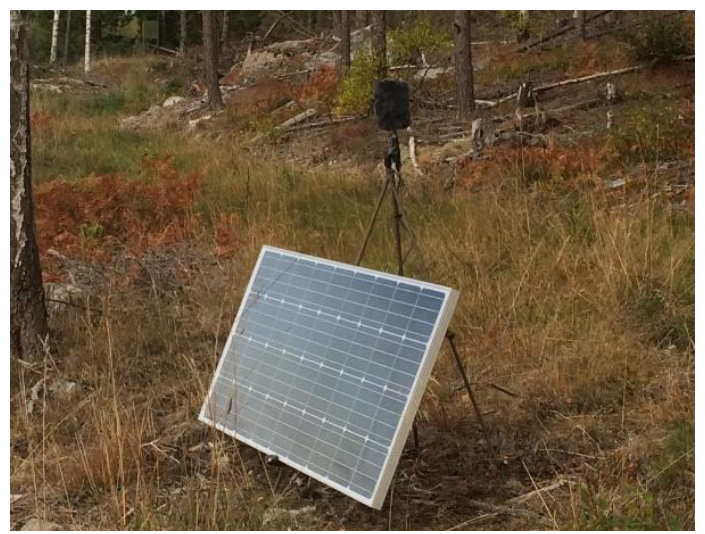

Figure 1: Photo of the acoustic measurement station. Depicted is the solar cell and the microphone with fitted windscreen

The core of the station is a SBC that processes, stores and uploads measurement data. Connected to the computer, through a sound card, is a microphone of type electretcondenser (the performance of the microphone is described in section 2.3.2 below). The microphone output signal is sampled at $48 \mathrm{kHz}$, with a bit-depth of 24 and the signal is filtered through 1/3-octave passbands, covering center frequencies from $25 \mathrm{~Hz}$ to $20 \mathrm{kHz}$. The slope and characteristics of the band-pass filters meets IEC 61672-1 standards for class-1 acoustical measurement equipment. From the output of each 1/3-octave band, A-weighted sound pressure levels are calculated and updated at $8 \mathrm{~Hz}$ intervals equivalent to the time-weighting "Fast". In addition to the $1 / 3$-octave band sound pressure levels, audio recordings are also performed to enable narrowband analysis and playback of single aircraft pass-by. The measurement station is powered by a solar panel and through GSM-connectivity measurement-data is uploaded to a central server for further compilation and analysis.

\subsubsection{Microphone}

The microphone used in the measurement station is a $6 \mathrm{~mm}$ electret-condenser microphone. In this particular microphone the preamplifier, soundcard and microphone capsule is built into one unit which connects directly to the computer via a USB-cable. Electret-condenser microphones are known for their robustness, linear response and cheap production costs. The noise-floor of the microphone and soundcard is estimated to be below $30 \mathrm{~dB}(\mathrm{~A})$ and the dynamic range is about $80 \mathrm{~dB}$. 


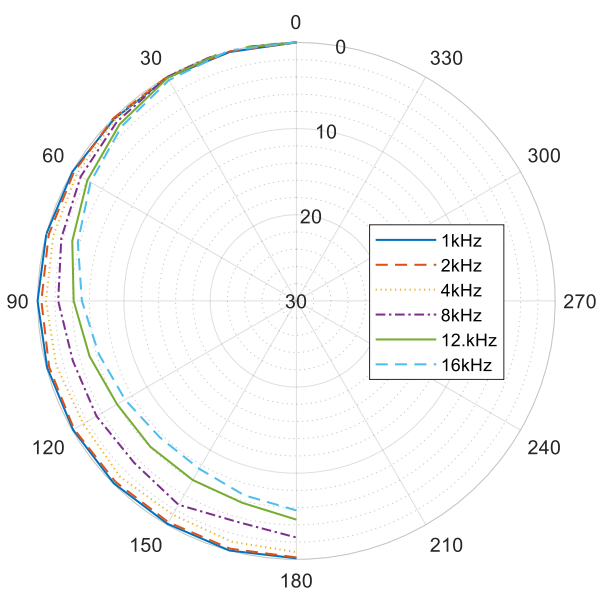

Figure 2: Polar plot of the microphones directivity for the 1/3-octave bands and center frequencies 1, 2, 4, 8, 12.5 and $16 \mathrm{kHz}$

In fig. 2, the directivity of the microphone in the 1/3-octave bands and center frequencies $1,2,4,8,12.5$ and $16 \mathrm{kHz}$ is shown. It can be seen that the response does not deviate more than $10 \mathrm{~dB}$ for any angle compared to the on-axis response. The directivity pattern of the microphone therefore meets the requirements according to the IEC 61672-1 standard for electro acoustic sound level meters. When acoustic measurements are made over long periods of time it is important that the performance of the microphone remains stable and that does not drift in sensitivity.

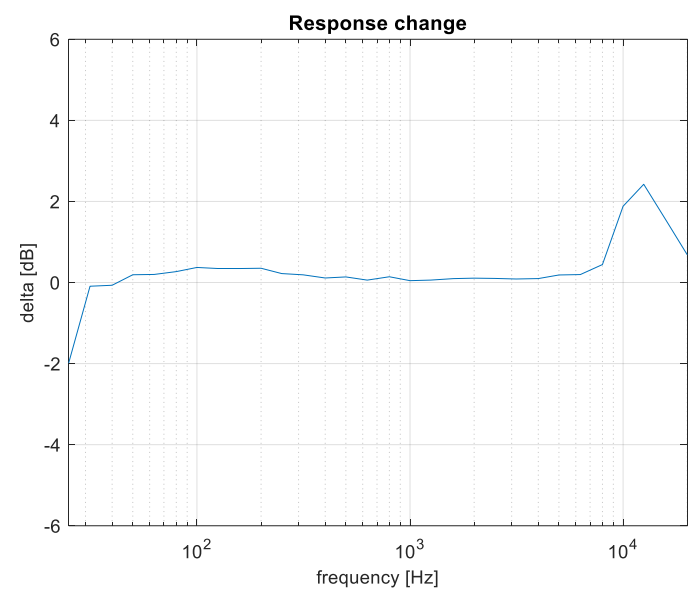

Figure 3: Change in frequency response of the microphone after 9 months of use in the field.

Figure 3. show the change in frequency response for a microphone used in the field for approximately 9 months. During this period, the weather changed from winter to summer, causing the microphone to be exposed to high temperatures and sub-zero degrees. However, as can be seen in figure 3 the response of the microphone remained essentially unchanged indicating that measurements taken over long periods produce reliable results. To mitigate windinduced noise the microphone is equipped with a windscreen, see fig. 1 . The windscreen, which is made of a foam core with a fur cover, also has a function as protection for rain and moisture. When fitting the windscreen to the microphone higher frequencies become attenuated. However, the characteristics of aircraft noise is only relevant up to $5 \mathrm{kHz}$, due to the air absorption of the relatively large measurement distances. Above $5 \mathrm{kHz}$ the sound absorption of the windscreen is insignificant. Rain and snow accumulating on the windscreen will likely change the acoustic properties and change the microphone's frequency response. As weather data is available, it is however possible to investigate statistically how the amount of precipitation affects the measurement data and then possibly omit measurements made under unfavorable weather conditions.

Several properties of the measurement equipment meet IEC 61672-1 class-1 standard, though the complete chain of components should not be considered a class-1 product. However, the authors consider the equipment to fulfill the purpose of survey measurements and to be adequate for the task of noise levels comparisons for different flights.

\subsubsection{Measurement arrangement}

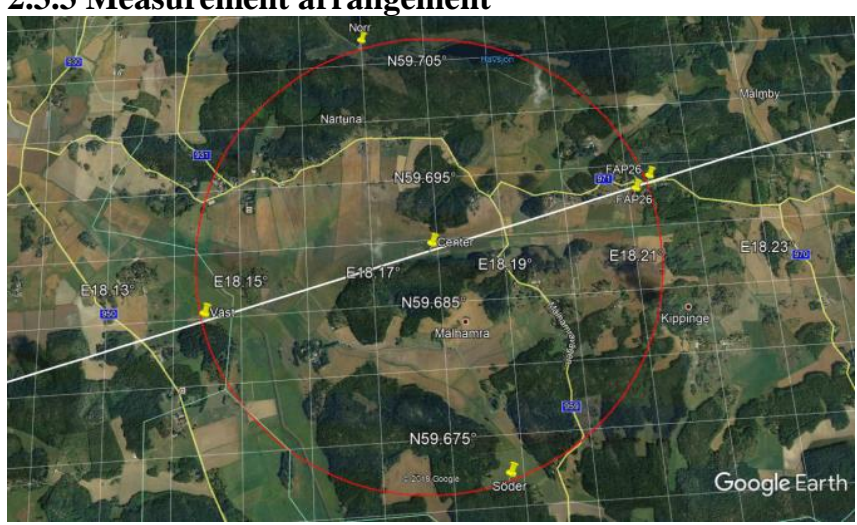

Figure 4: Positions of the measurement stations. The red circle has a radius of $2 \mathrm{~km}$. The white line shows the approach path to runway $26 R$.

At an initial phase of the measurement campaign 5 measurement stations where placed beneath the final approach path to runway $26 \mathrm{R}$ to cover the sound propagation in lateral and longitudinal directions, see fig. 4. The most east bound station, is positioned beneath the Final Approach Point (FAP), where every aircraft approaching runway $26 \mathrm{R}$ have to pass to enter the 3-degree glide-path down to the runway. The center and west station are positioned further down the glide path and spaced $2 \mathrm{~km}$ apart. The north and south station are positioned perpendicular to the glide path and $2 \mathrm{~km}$ out from the center station. The spacing and location of the measurement stations was chosen because it is at this distance and altitude the pilots usually deploy landing gear and flaps, which both are two major sources of air frame noise. The microphones on the measurement stations are positioned 1.2 meters above ground.

\section{Results and Discussion}

In the following section a snap-shot of noise measurement data will be presented that highlight how different pass-by noise levels can be generated by the same individual aircraft and for a seemingly similar approach route. The 
measurements where made for approaching aircrafts to runway 26R at Arlanda airport during the period May to June2019 and are presented in pairs where each pair show two pass-by events of the same aircraft. The events of the individual aircraft occurred during the same day and sometimes separated only by a few hours. In figures 5-10, in addition to noise levels recorded by the five measurement stations, ADB-S data for the geographical-altitude and velocity of the aircraft is also shown. The altitude is given in meters while the velocity is given as meters per second $(\mathrm{m} / \mathrm{s})$. (Perhaps an unorthodox quantity in the context of airplanes, but it facilitated the numbering properties of the figure axis. $1 \mathrm{~m} / \mathrm{s}=1.94384449$ knots). All three aircrafts belong to the SAS fleet and are of the type: Boeing 737, Airbus A321 and A320.

\subsection{Airbus A320}

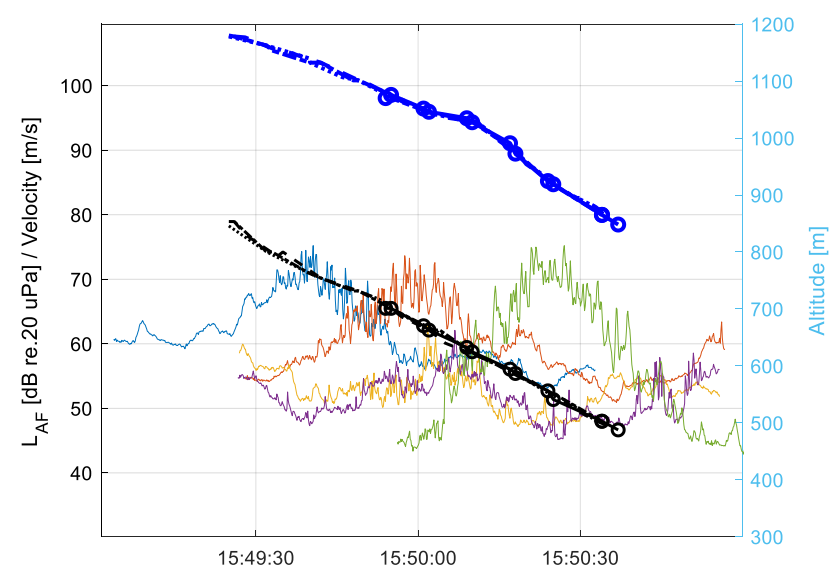

Figure 1: First pass-by noise of the Airbus A320 at 15:49 flying over the measurement area. The blur thin right-most line shows the $L_{A F}$ measured at the FAP-station. The red line is the Center-station and the green line is the West-station. Blue thick-dotted line shows the airplanes velocity in $\mathrm{m} / \mathrm{s}$ while the black thick-dotted line shows the altitude in meters.

Figure 5 show noise level of the first pass-by of the Airbus A320 occurring at 15:49. The wind conditions at this time of the day was $4 \mathrm{~m} / \mathrm{s}$, heading 200 degrees. We can clearly see the noise level build up and the following level decrease as the aircraft pass over the measurement positions, creating characteristic noise contours resembling those of small hills. The most pronounced contours are of course seen for the stations directly under the flight path, were the distance: source-receiver is the smallest, i.e. at the FAP, Center and West station. For this particular pass-by, the noise levels reach about $70 \mathrm{~dB}(\mathrm{~A})$, which would sort into the category: "high noise level pass-by". One could expect that the noise level on ground would increase as the aircraft drops in altitude and the source-receiver distance shrinks, in this case by over 200 meters from the FAP to the West station. However, no such effect can be observed as the contours for all three stations have a similar shape. The effect of noise-power distance is anyhow well observed for the lateral stations where the noise level only reaches a maximum of about 60 $\mathrm{dB}$ or lower. The blue thick dotted line shows the velocity and we can see that it is steady decreasing from $110 \mathrm{~m} / \mathrm{s}$ in the start of the pass-by to below $80 \mathrm{~m} / \mathrm{s}$ in the final stretch, with an almost constant deacceleration. From the collection of pass-by measurements this kind of "high noise level passby" was rather common for the Airbus A320.

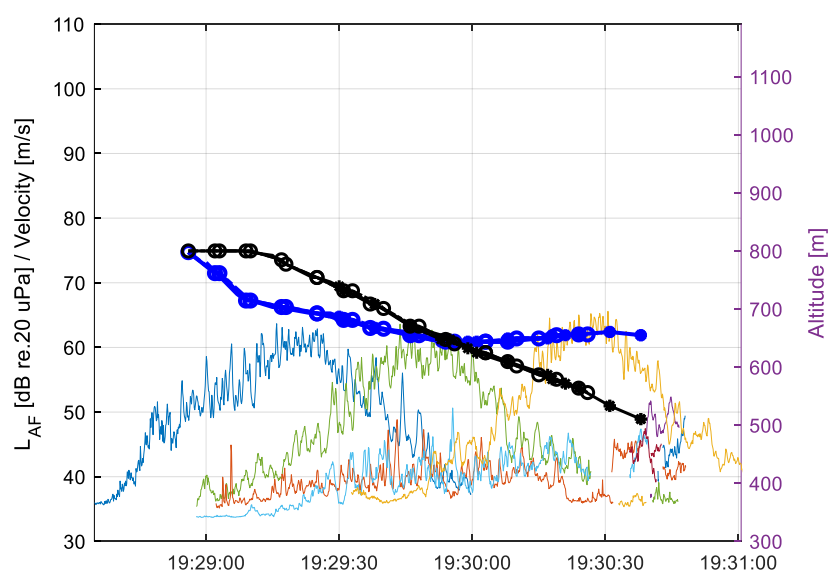

Figure 2: Second pass-by noise of the Airbus A320 at 19:29 flying over the measurement area. The blur thin right-most line shows the $L_{A F}$ measured at the FAP-station. The green line is the Center-station and the yellow line is the Weststation. Blue thick-dotted line shows the airplanes velocity in $\mathrm{m} / \mathrm{s}$ while the black thick-dotted line shows the altitude in meters.

Figure 6 shows the second pass-by of the A320 at 19:29 later the same day. The wind was $4 \mathrm{~m} / \mathrm{s}$ but the direction had slightly changed to 180 degrees heading. The noise levels generated by this pass-by is less common and would sort into the category: "low noise level pass-by". Compared to the previous result, there are some major differences. The noise levels are about $10 \mathrm{~dB}$ lower, which is a notable difference considering that it takes an eight times more powerful noise source to generate an increase of $9 \mathrm{~dB}$. The other major difference is the reduced velocity for with which the aircraft travels. The velocity is also more constant, suggesting a smoother approach with less deaccelerating. The initial velocity is about $75 \mathrm{~m} / \mathrm{s}$ which quickly drops to $65 \mathrm{~m} / \mathrm{s}$, before the aircraft reaches the FAP. The velocity then decreases slowly to $60 \mathrm{~m} / \mathrm{s}$ and is almost constant for the reminder of the pass-by. Compared to the earlier pass-by the velocity is reduced by over one third for major parts of the glide path. The difference in noise levels and velocity profiles of these two flights, which were performed by the same aircraft, strongly suggest the obvious correlation between velocity and generated noise level.

\subsection{Boeing 737}




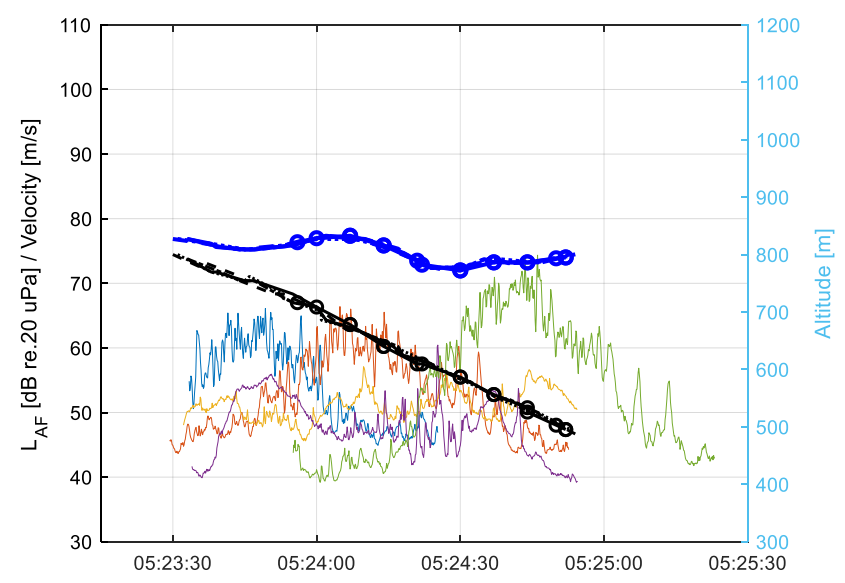

Figure 3: First pass-by noise of the Boeing 737at 05:23 flying over the measurement area. The blur thin right-most line shows the $L_{A F}$ measured at the FAP-station. The red line is the Center-station and the green line is the West-station. Blue thick-dotted line shows the airplanes velocity in $\mathrm{m} / \mathrm{s}$ while the black thick-dotted line shows the altitude in meters.

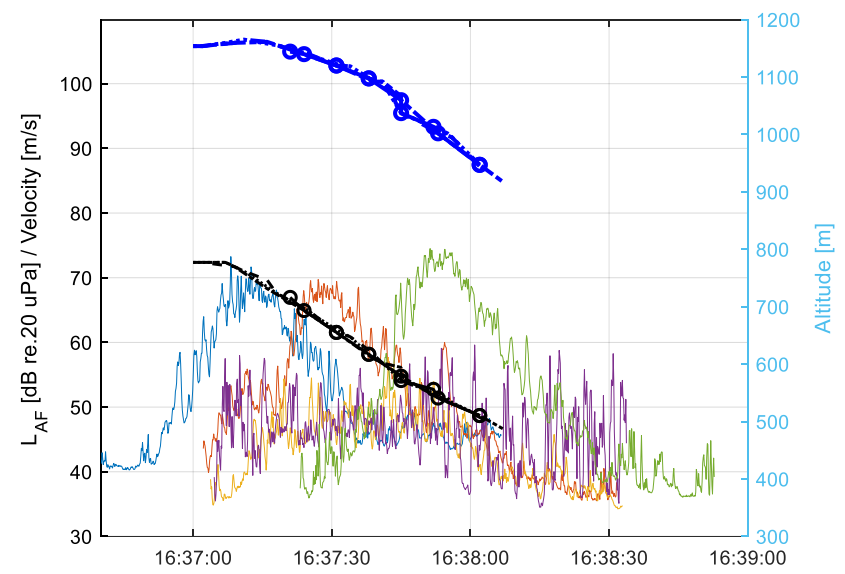

Figure 4: Second pass-by noise of the Boeing 737 at 16:37 flying over the measurement area. The blur thin right-most line shows the $L_{A F}$ measured at the FAP-station. The red line is the Center-station and the green line is the West-station. Blue thick-dotted line shows the airplanes velocity in $\mathrm{m} / \mathrm{s}$ while the black thick-dotted line shows the altitude in meters.

Figure 7 and 8 show a similar result for one Boeing 737, though not as drastic as for the A320. These two pass-by's occurred at 05:23 and 16:37. The wind this day was $5 \mathrm{~m} / \mathrm{s}$ in the morning with direction 250 degrees and $3 \mathrm{~m} / \mathrm{s}$ in the evening with direction 240 degrees. For these two pass-by measurements the noise level difference is about $5 \mathrm{~dB}$. The velocity difference is also less but the acceleration for the low and high noise level pass-by's is similar to previous results. Note that West noise contour (the right-most curve) in fig.78 , is higher than the other contours, showing the effect of noise-power distance.

\subsection{Airbus A321}

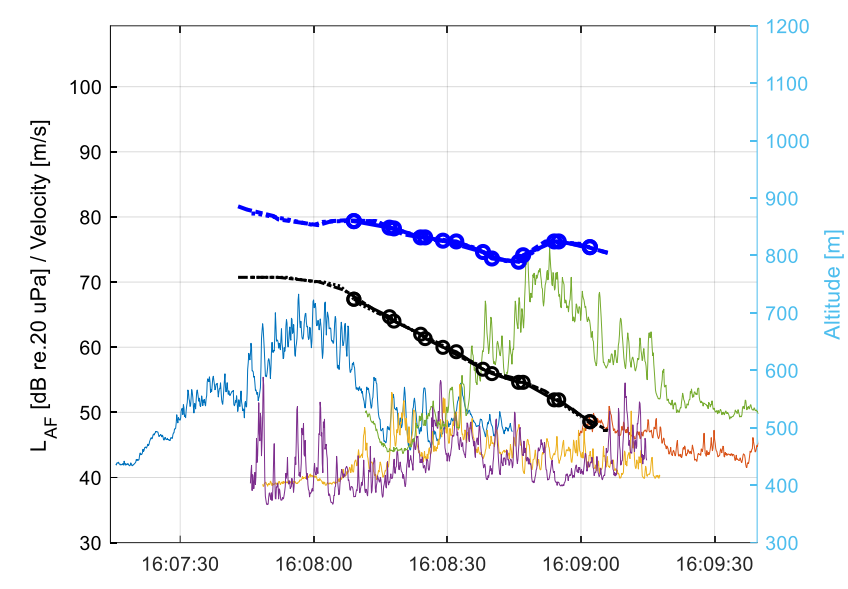

Figure 5: First pass-by noise of the Airbus A321 at 16:07 flying over the measurement area. The blur thin right-most line shows the $L_{A F}$ measured at the FAP-station. The green line is the West-station and blue thick-dotted line shows the airplanes velocity in $\mathrm{m} / \mathrm{s}$ while the black thick-dotted line shows the altitude in meters.

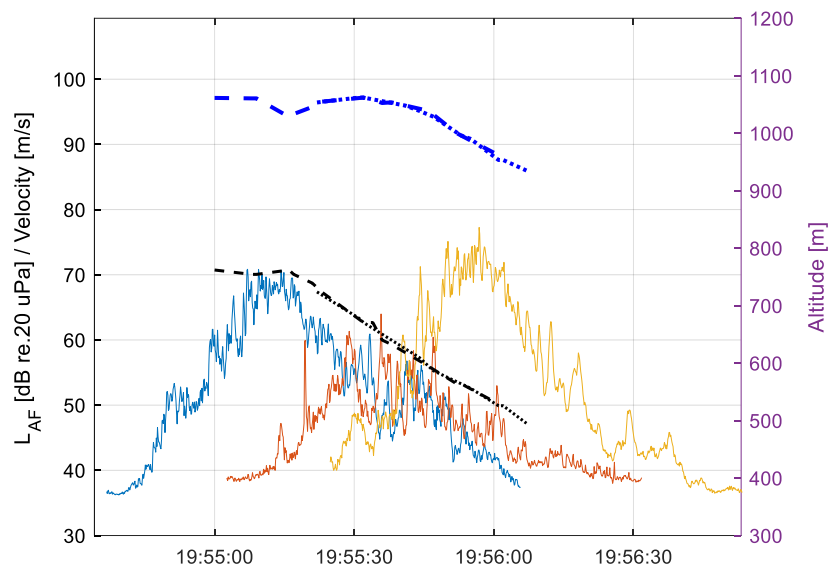

Figure 6: Second pass-by noise of the Airbus A321 at 19:55 flying over the measurement area. The blur thin right-most line shows the $L_{A F}$ measured at the FAP-station. The yellow line is the West-station and blue thick-dotted line shows the airplanes velocity in $\mathrm{m} / \mathrm{s}$ while the black thick-dotted line shows the altitude in meters.

Figure 9 and 10 show a pair of additional measurements, this time for one Airbus A321 passing over the measurement area at 16:08 and 19:55. The wind condition for the earlier flight was $4 \mathrm{~m} / \mathrm{s}$ with direction 300 degrees and $3 \mathrm{~m} / \mathrm{s}$ for the later flight with direction 310 degrees. During this day the Center station was out of order leaving measurements only for the FAP and West station. Comparing the two measurements in fig. 9-10 we can see that it is about a $5 \mathrm{~dB}$ noise level difference between the flights and that it is the later flight that generates the highest noise level. The difference is mostly pronounced in the noise contour of the FAP station. A reason for the higher noise level at the West station could be that the landing gear or flaps are deployed. ADB-S data does not give any parameters for flaps or landing gear deployment. However, an indication of alternations in flight configuration could perhaps be seen in the flights velocity as sudden 
changes. Maybe this is what is seen in fig. 9 at 16:08:45? However, to verify such a statement ADB-S data would have to be run against FDR-data or other records of flight parameters, such as visual observations.

\subsection{Further work}

The above snap-shots in fig. 5-10 are taken from a batch of measurements containing about 100 passages of the A320, c. 40 of the Boeing 737 and 20 for the A321. No statistical calculations have been performed on the material and the estimates are to be considered as rough. The continued measurement analysis will contain a statistical analysis that also include a greater number of aircraft pass-by's. It is thus necessary to consider the above result only as preliminary observations. To minimize the number of error sources the comparison of pass-by noise levels have been restricted to flights of the same individual aircraft and to flights that occurred in close proximity. There are however, a number of parameters that can have changed during the time of the two passby's. One factor for instance, is the passenger load which greatly would influence the thrust-settings and directly alter the strength of the noise source. None the less, the large differences in generated noise levels does raise some questions. Is it the airframe or engine that dominates the noise? How does the duration of the pass-by influence the noise perception, is it better to have a slow and quiet pass-by than a fast and loud? These are questions that have to be answered in the continued work of this project in investigating the possibilities of optimizing flight procedures for a quieter approach

\section{Conclusion}

A system of acoustic measurement stations has been built and deployed in the field east of runway 26R at Arlanda airport to investigate how different aircraft operational procedures impact the noise distribution on ground. Together with measurements of the single aircraft pass-by noise at ground level ADB-S data of the flights trajectory have been recorded for a prolonged period of time. This paper presents measurements that compare the noise level on ground generated by the same individual aircraft at different occasions during the same day, for similar flight trajectories and meteorological conditions. The differences in generated noise levels was found to be as large as $10 \mathrm{~dB}$. A reduced velocity for the less noisy flight, indicate that this is the main factor that govern noise generation. Velocity differences were as large as 90 knots. Although, the result of this paper is only preliminary and a first step in the analysis of the noise situation, the large discrepancies in noise emission shows possibilities to optimize for a quieter approach procedure.

\section{References}

[1] Community Oriented Solutions to Minimize Aircraft noise annoyance (COSMA), EU/FP7 Project reference: 234118, http://www.fp7-cosma.eu/

[2] Graham W. R., Hall C. A. och Vera Morales M., (2014) "The potential of future aircraft technology for noise and pollutant emissions reduction," Transport Policy, 34, 36 $-51$

[3] Miedema, H. M. och Oudshoorn, C. G. (2001). Annoyance from transportation noise: relationships with exposure metrics DNL and DENL and their confidence intervals. Environmental Health Perspectives, 109(4), 409-416.

[4] Webpage: https://opensky-network.org, Last visited 2019-07-30

[5] Webpage: https://www.smhi.se/data/utforskaren-oppnadata/meteorologisk-modell-arome-prognosdata-realtid, Last visited 2019-07-30 